\title{
Christian Grawe: Veröffentlichungen über Theodor Fontane
}

\section{Selbständige Schriften}

Führer durch die Romane Theodor Fontanes. Ein Lexikon der Personen, Schauplätze und Kunstwerke. Frankfurt/M.-Berlin-Wien: Ullstein 1980.

Zweite, veränderte und erweiterte Auflage unter dem Titel Führer durch Fontanes Romane. Stuttgart: Reclam 1995.

Theodor Fontane »Effi Briest«. Frankfurt/M.: Diesterweg ${ }^{7} 1998$ (1985).

Fontane-Chronik. Mit 12 Abbildungen. Stuttgart: Reclam 1998.

\section{Editionen}

Theodor Fontane »Cécile». Mit Anmerkungen und Nachwort hg. von Christian Grawe. Stuttgart: Reclam 1982.

(Der zwölfte Aufsatz des vorliegenden Bandes)

Theodor Fontane »Meine Kinderjahre". Mit Nachwort und Anmerkungen hg. von Chrstian Grawe. Stuttgart: Reclam 1986.

(Der sechzehnte Aufsatz des vorliegenden Bandes)

Theodor Fontane. Romane und Novellen - Interpretationen. Hg. von Christian Grawe. Stuttgart: Reclam 1990.

(Beiträge von zehn Forschern aus fünf Ländern)

Alles kommt auf die Beleuchtung an."Fontane zum Vergnügen. Mit 7 Illustrationen. Hg. von Christian Grawe. Stuttgart: Reclam 1994.

Theodor-Fontane-Handbuch. Hg. von Christian Grawe and Helmuth Nürnberger. Stuttgart: Alfred Kröner 1999.

(Beiträge von 17 Forschern aus sechs Ländern)

\section{Buchbeiträge}

"Fontanes neues Sprachbewußtsein in Der Stechlin«. In: Christian Grawe, Sprache im Prosawerk. Bonn: Bouvier ${ }^{2} 1987$ (1974), S. 38-62.

(Der neunzehnte Aufsatz des vorliegenden Bandes) 
»Von Krieg und Kriegsgeschrei. Fontanes Kriegsdarstellungen im Kontext«. In: Theodor Fontane im literarischen Leben seiner Zeit. Beiträge zur FontaneKonferenz vom 17. bis 20. Juni 1986 in Potsdam. Berlin: Deutsche Staatsbibliothek 1987, S. 67-106.

(Der dritte Aufsatz des vorliegenden Bandes)

»Theodor Fontane«. In: Deutsche Dichter. Leben und Werk deutschsprachiger Autoren. Band 6: Realismus, Naturalismus und Jugendstil. Stuttgart: Reclam 1989, S. 126-151.

»Preußen 1803 bis 1813 im >vaterländischen Roman ‘: Willibald Alexis, George Hesekiel, Theodor Fontane «. In: Literatur und Geschichte 1788-1988. Hg. von Gerhard Schulz and Tim Mehigan in Zusammenarbeit mit Marion Adams. Bern u. a.: Peter Lang 1989, S. 141-179.

(Der sechste Aufsatz des vorliegenden Bandes)

"Quitt - Lehnert Menz zwischen Todesverfallenheit und Auferstehung«. In: Theodor Fontane. Romane und Novellen - Interpretationen. Hg. von Christian Grawe. Stuttgart: Reclam 1990, S. 157-184.

(Der vierzehnte Aufsatz im vorliegenden Band)

»Effi Briest - Geducktes Vögelchen in Schneelandschaft: Effi von Innstetten, geborene von Briest «. In: Theodor Fontane. Romane und Novellen - Interpretationen. Hg. von Christian Grawe. Stuttgart: Reclam 1990, S. 217-242.

(Der achtzehnte Aufsatz des vorliegenden Bandes)

Die Artikel über Theodor Fontane:

Wanderungen durch die Mark Brandenburg - Vor dem Sturm - Grete Minde Ellernklipp - L'Adultera - Schach von Wuthenow - Graf Petöfy - Unterm Birnbaum - Cécile - Irrungen, Wirrungen - Stine - Quitt - Unwiederbringlich Frau Jenny Treibel - Effi Briest - Die Poggenpuhls - Der Stechlin - Mathilde Möhring. In: Reclams Romanlexikon. Band 2: Von der Romantik bis zum Naturalismus. Hg. von Frank Rainer Max und Christine Ruhrberg. Stuttgart: Reclam 1999, S. 353-378.

Der Fontanesche Roman, Vor dem Sturm (der zehnte Aufsatz des vorliegenden Bandes) - Grete Minde - Ellernklipp - L'Adultera - Schach von Wuthenow - Graf Petöfy - Irrungen, Wirrungen - Quitt - Unwiederbringlich - Frau Jenny Treibel. In: Theodor-Fontane-Handbuch. Hg. von Christian Grawe und Helmuth Nürnberger. Stuttgart: Alfred Kröner 1998, S. 466-554, 575-627. 
") Mit 78 ist man ein unsicherer Passagier<: Fontanes letztes Jahr an der Schwelle des 20. Jahrhunderts«. In: Theodorus victor. Theodor Fontane. Der Schriftsteller des 19. am Ende des 20. Jahrhunderts. Eine Sammlung von Beiträgen. Hg. von Roland Berbig. Frankfurt am Main u. a.: Peter Lang 1999, S. 221-241. (= Literatur - Sprache - Region Band 3)

(Der neunte Aufsatz des vorliegenden Bandes)

") Une saison en enfer $:$ Die erste Saison der Freien Bühne und Fontanes Kritiken«. In: Theodor Fontane. Am Ende des Jahrhunderts. Internationales Symposium des Theodor-Fontane-Archivs zum 100. Todestag Theodor Fontanes, 13.-17. September 1998 in Potsdam, Band III. Hg. von Hanna Delf von Wolzogen in Zusammenarbeit mit Helmuth Nürnberger. Würzburg: Königshausen \& Neumann 1999, S. 267-283.

(Der siebte Aufsatz des vorliegenden Bandes)

"Ein ausgeschiedenes und wieder aufgefundenes Kapitel aus Der Stechlin«. In: For she' a Jolli good fellow. Charlotte Jolles zum Geburtstag. Hg. von Manfred Horlitz. Flensburg: Baltica-Verlag 1999, S. 60-65.

»)Italian Hours «: Theodor Fontane und Henry James in Italien in den 1870er Jahren«. In: Fontane und die Fremde, Fontane und Europa, hg. von Konrad Ehlich. Würzburg: Königshausen \& Neumann 2002, S. 276-294.

(Der fünfte Aufsatz des vorliegenden Bandes)

\section{Aufsätze}

"Wuthenow oder Venedig. Analyse von Schachs Reisefantasie im Fontaneschen Kontext«. In: Wirkendes Wort 30/4 (1980), S. 258-267.

(Der elfte Aufsatz des vorliegenden Bandes)

„Käthe von Sellenthins Irrungen, Wirrungen«. In: Fontane Blätter, H. 34 (1982), S. 84-100.

(Der dreizehnte Aufsatz des vorliegenden Bandes)

„Crampas und sein Lieblingsdichter Heine und einige damit verbundene Motive«. In: Jahrbuch der Raabe-Gesellschaft 1982, S. 142-164.

(Der siebzehnte Aufsatz des vorliegenden Bandes)

"Lieutenant Vogelsang a. C. und Mr. Nelson aus Liverpool: Treibels politische und Corinnas private Verirrungen in Frau Jenny Treibel«. In: Fontane Blätter, H. 38 (1984), S. 588-606.

(Der fünfzehnte Aufsatz des vorliegenden Bandes) 
»Of Bildungsromane and Zeitromane. Some Books about the 19th Centrury German Novel«. (Sammelrenzension von 8 Büchern, 4 davon über Fontane). In: AUMLA, no. 69 (1988), S. 193-205.

»Über die Sinnentleerung der Literatur. Polemische Anmerkungen zu Bernd W. Seilers Effi Briest-Aufsatz« in Der Deutschunterricht H. 104. In: Diskussion Deutsch, H. 106 (1989), S. 208-211.

»)Es schlug gerade ...<. Zur Gestaltung eines Zeitelements in Theodor Fontanes Romanen«. In: Fontane Blätter, H. 51 (1991), S. 141-156.

„Warum Fontane kein Barbarossa-Epos schrieb und andere Vermutungen über den mittleren Fontane«. In: Fontane Blätter, H. 58 (1994), S. 270-295. (Der erste Aufsatz des vorliegenden Bandes)

»Einen frischen Trunk Schiller zu tun 1870-1889« (1. Teil). In: Fontane Blätter, H. 62 (1996), S. 76-87.

(2. Teil). In: Fontane Blätter, H. 63 (1997), S. 66-90.

(Der vierte Aufsatz des vorliegenden Bandes)

»Die wahre hohe Schule der Zweideutigkeit<. Frivolität und ihre autobiographische Dimension in Fontanes Romanen«. In: Fontane Blätter, H. 65/66 (1998), S. 138-162.

(Der achte Aufsatz des vorliegenden Bandes) 